\title{
La Historia de la Comunicación: aportaciones a la construcción de un humanismo plural, posicionado, en red y cooperativo
}

\author{
Amparo MOREnO SARDÀ \\ Universidad Autónoma de Barcelona \\ amparo.moreno@uab.es
}

\begin{abstract}
Resumen:
Las TTIICC favorecen un cambio profundo en la construcción del conocimiento sobre el pasado y el presente de la existencia humana, al facilitar la participación de mujeres y hombres. Pero para ello es necesaria también una revolución de las Ciencias Humanas y Sociales que modifique un paradigma androcéntrico, que los considera objeto de estudio y menosprecia las aportaciones personales como insignificantes. La Historia de la Comunicación, al proponerse articular las redes de comunicación tejidas en los itinerarios personales con las tramas históricas colectivas, hace aportaciones a este cambio.
\end{abstract}

Palabras clave: Historia de la Comunicación; Humanismo plural posicionado, en red y cooperativo; Revolución científica.

\section{History of Communication: Contributions to the Construction of a Cooperative Plural Humanism, Well Positioned and Networking Active.}

\begin{abstract}
:
Facilitating the participation of women and men, ICTs can ensure a profound change in the construction of the past and present knowledge of human existence. However, for this, it is necessary a revolution of the Human and Social Sciences that will modify an androcentric paradigm that considers people as a study object and underestimates their personal contributions. The History of Communication makes contributions to this change because it proposes to articulate the personal networking communication and life plans of individual people with the collective history.
\end{abstract}

Key Words: History of Communication; Cooperative Plural Humanism; Well Positioned and Networking Active; Scientific revolution

\section{Referencia normalizada:}

Moreno Sardà, A. (2014): La historia de la comunicación: aportaciones a la construcción de un humanismo plural, posicionado, en red y cooperativo. Historia y Comunicación Social. Vol. 19. Núm. Especial Febrero. Págs. 79-91.

Sumario: Presentación. 1. La necesaria revolución de las ciencias humanas y sociales: los seres humanos, de 'objeto de estudio' a sujetos activos en la construcción de un humanismo plural. 2. La Historia de la Comunicación: necesidad de un nuevo paradigma para comprender las sociedades contemporáneas. 2.1. La Historia de la Comunicación: contexto histórico. 2.2. Limitaciones de las humanidades y las ciencias sociales: el orden androcéntrico del discurso académico. 2.3. La actividad humana como actividad comunicativa: paradigmas para una Historia de la Comunicación no-androcéntrica. 3. De las propuestas para la innovación docente a los instrumentos para la participación ciudadana en la cons- 
trucción de un humanismo plural. 3.1. Metodología. 3.2. Dos ejemplos. 4. Conclusiones. Referencias bibliográficas.

Las TTIICC están favoreciendo un cambio profundo en los procedimientos de construcción de conocimiento sobre el pasado y el presente de la existencia humana. Tradicionalmente, el conocimiento ha sido valorado significativo cuando lo han producido minorías vinculadas al poder, mientras que el que elabora cualquier mujer u hombre a partir de su experiencia cotidiana ha sido menospreciado como si no fuera significativo para comprender la existencia humana. Las TTIICC facilitan hoy que cualquier persona haga aportaciones para construir un conocimiento colectivo. Sin embargo, no es suficiente con la tecnología. Es imprescindible una revolución en las ciencias humanas y sociales: que los profesionales hagan autocrítica del paradigma académico dominante, androcéntrico, restrictivo y parcial, que alimenta explicaciones incapaces de dar cuenta de los cambios que se han producido en las sociedades contemporáneas, y que adopten un nuevo enfoque que abarque la pluralidad humana de la sociedad global. Sólo así podrán colaborar en la elaboración de un humanismo plural.

En este artículo explicamos cómo la tarea de formular la Historia de la Comunicación en la Facultad de Ciencias de la Comunicación de la Universidad Autónoma de Barcelona, en los años 70 del siglo pasado, nos planteó una serie de problemas que nos condujeron a explorar soluciones que coinciden con las que reclaman hoy las TTIICC para facilitar la participación de la ciudadanía plural en la construcción de un humanismo plural; y exponemos la metodología que puede facilitar esta tarea, y dos ejemplos en los que la aplicamos.

\section{La necesaria revolución de las ciencias humanas y sociales: los seres humanos, de "objeto de estudio" a sujeros activos en la construcción de un humanismo plural}

Utilizamos la noción "revolución cientifica” en el sentido que le dio el historiador y filósofo de la ciencia Thomas Samuel Kuhn. Kuhn explica que la "ciencia normal”, la actividad que realizan habitualmente los científicos, parte de un paradigma compartido que se ocupa de resolver "enigmas" sin cuestionarse ese paradigma. Sin embargo, en algún momento, algunos científicos perciben unos problemas que no se pueden resolver en el marco del paradigma aceptado. Estos problemas aparecen como una "anomalia". Los científicos que se aferran al paradigma aceptado menosprecian esta "anomalía”. Por el contrario, aquellos que la han detectado y consideran necesario tenerla en cuenta, dedican un periodo más o menos prolongado a explorar la "zona de anomalía" hasta que consiguen producir un ajuste de la teoría que les lleva a ver la naturaleza de otra manera, y a formular un nuevo paradigma para inter- 
pretarla y poder asumir como "normal" lo que se había considerado "anómalo". Durante este proceso, se producen confrontaciones entre los que continúan fieles al paradigma anterior y los que exploran las "zonas de anomalía" y formulan nuevos paradigmas y nuevas explicaciones (Kuhn: 1962).

Sostenemos la hipótesis de que las Ciencias Humanas y Sociales han de resolver hoy algunas "anomalias" que ponen de manifiesto realidades sociales que antes los profesionales no habían tenido en cuenta y a las ahora han de atender, debido a las transformaciones que se han producido y afectan también a la propia Universidad, transformaciones a las que los recursos técnicos están dando respuesta.

Hoy, gracias a Internet, el conocimiento sobre la existencia humana y el funcionamiento social ya no es patrimonio exclusivo de profesionales especializados que integran academias a las que se accede por procedimientos restrictivos; se puede enriquecer con las aportaciones de cualquier persona. Pero no es suficiente con las aportaciones individuales. Tampoco basta con los recursos técnicos. Es necesario también que el conocimiento experto de las Ciencias Humanas y Sociales se transforme para que pueda colaborar a que los relatos personales dejen de tener un valor anecdótico, insignificante, marginal, y cobren sentido y enriquezcan un humanismo plural, polifónico, expresión de las sociedades actuales. La dificultad principal estriba en que para ello los expertos han de desaprender el paradigma tradicional, que considera a los seres humanos como "objeto de estudio" pasivo, y los reconozcan como sujetos activos. Y como advirtió Aristóteles, "es más dificil olvidar lo aprendido que aprender por primera vez” (Moreno Sardà: 1988).

En esta tarea reclamamos un papel para la Historia de la Comunicación como la hemos formulado a partir de los años 70 del siglo pasado en la Facultad de Ciencias de la Comunicación de la Universidad Autónoma de Barcelona, con rasgos específicos, porque es el resultado de haber buscado las aportaciones de las Humanidades y las Ciencias Sociales, haber tropezado con importantes limitaciones, haber explorado "zonas de anomalia", haber formulado nuevos paradigmas para considerarlas normales, y haberlos puesto a prueba con propuestas de innovación docente.

\section{La historia de la comunicación: necesidad de un nuevo paradigma para comprender las sociedades contemporáneas}

\subsection{La historia de la comunicación: contexto histórico}

La Historia de la Comunicación es una disciplina joven como lo es el objeto de estudio en el que se centra, los medios de comunicación de masas que están en la base de la cultura de masas y la sociedad de la información, y no existe acuerdo sobre su objeto de estudio debido especialmente a los condicionantes con que se encuentra, que provienen de las Ciencias Humanas y Sociales de las que se nutre (Moreno Sardà: 1997). 
En sus inicios, la Historia de la Comunicación se centró en el que fue cronológicamente el primero de los medios, la prensa escrita, y se interesó por las biografías de los grandes editores, las instituciones específicas y la catalogación de las cabeceras, interés al que todavía hoy se limitan algunos estudiosos (Rowland: 1997). Pero la aparición de nuevos medios para responder a los cambios sociales ha obligado a modificar este enfoque.

Las primeras historias del periodismo coincidieron con el papel que alcanzó desde mediados del siglo XIX en los Estados nacionales europeos, en los que se fue convirtiendo en un medio de comunicación de masas. El interés creció en las primeras décadas del siglo XX, cuando se desarrolló el periodismo como profesión y los centros de formación de periodistas. Y a medida que se implantaron otros medios se elaboraron también las correspondientes historias.

El papel del periodismo estuvo relacionado con un conjunto de transformaciones históricas vinculadas a la expansión imperialista de los Estados europeos que se repartieron el mundo (Barraclough: 1965; Kennedy: 2006) y construyeron los mercados financieros controlados por la Bolsa (Flichy: 1991). En este contexto, la revolución industrial impulsó nuevas redes y medios de transporte - ferrocarriles y barcos a vapor - y de comunicación - telégrafo y cables submarinos - que tejió un sistema neurálgico que cruzó océanos y continentes, permitió alcanzar territorios cada vez más amplios en menos tiempo (Moreno Sardà: 1986b), y generó la noción de periodismo diario y actualidad (Carey: 1997) vigente hasta hace muy poco. En este proceso desempeñaron un papel decisivo las Agencias de Noticias que desde el principio se organizaron de acuerdo con los intereses imperialistas de los Estados y colaboraron en sus confrontaciones y acuerdos por el "reparto del mundo".

Esta expansión, que desembocó en la primera Guerra Mundial, generó también profundas transformaciones en el interior de los estados que se manifestaron en el funcionamiento político - implantación del definido como "sufragio universal" durante mucho tiempo limitado a los "pater familiae" -, en la actividad económica, en los conflictos sociales, en la modernización de la vida cotidiana que se introducía especialmente entre las familias acomodadas de las ciudades, en la cultura. De todos ellos, característicos de lo que se llamó la "sociedad de masas", dio cuenta el periodismo y la publicidad comercial que lo financio que, junto con la fotografía, incorporaron a la cultura letrada un universo simbólico icónico.

Los conflictos entre las minorías y las mayorías que reclamaban derechos sociales y políticos, y entre los estados europeos por el dominio del mundo, se acentuaron con los fascismos y derivaron en una Segunda Guerra Mundial, situación en la que los medios de comunicación de masas se utilizaron ampliamente como instrumentos de propaganda.

El nuevo orden que se implantó tras la Segunda Guerra Mundial, conocido como "Guerra Fría", estuvo acompañado de cambios fundamentales en los sistemas de transporte y de comunicación. Además de la prensa, el cine y la radio, se introdujo la televisión, proliferaron los medios de reproducción de sonidos y de imágenes y 
se pusieron al alcance de 'las masas', en los hogares y gracias a los transistores en unos medios de transporte que facilitaron amplias movilizaciones de personas y de bienes por razones políticas, económicas y de ocio. Y estos medios, financiados en buena parte por una publicidad comercial que promovía la sociedad de consumo entre amplios sectores sociales, reprodujeron y difundieron el universo material y simbólico conocido como "cultura de masas".

De este modo, a partir de los años 50 y 60 resultó imprescindible hacer enfoques globales como los de Harold Innis en Empire and Communications (1950) y en The Bias of Communication (1952); Marshall Mc Luhan en La comprensión de los medios como extensiones del hombre; y Jurgen Habermas en Historia y crítica de la opinión pública, obras publicadas en 1962. A partir de los años 70 se empezó a debatir sobre la Historia de la Comunicación, los interrogantes a los que debe responder y las relaciones entre medios de comunicación, transformaciones sociales y repercusiones de los medios en las formas de conocimiento. Desde entonces, el incremento de obras pone de manifiesto la consolidación y el reconocimiento de la Historia de la Comunicación como disciplina académica $\mathrm{y}$, aunque coexisten enfoques diversos, esta disciplina ha tenido que ampliar los enfoques tradicionales, centrados en los medios de comunicación y en los estados nacionales, para dar cuenta de una realidad social amplia y compleja en la que se interconectan las prácticas cotidianas de las personas en un mundo globalizado (McBride y otros: 1980; McNeill y McNeill: 2010).

Recordemos también el papel decisivo que ha tenido la Cibernética (Wiener: 1965), a partir de la industria aeroespacial, y em las redes de comunicación e información que articulan Internet y están en la base de la "sociedad de la Información" (Castells: 2001).

La Historia de la Comunicación, para dar cuenta de estos cambios y también de las permanencias, ha tenido que resolver problemas derivados del paradigma "normal" de las Humanidades y las Ciencias Sociales.

2.2. Limitaciones de las humanidades y las ciencias sociales: el orden androcéntrico del discurso académico

En nuestro caso, los problemas aparecieron en cuanto nos propusimos elaborar un relato de la Historia de la Comunicación para la docencia, y al investigar sobre las representaciones simbólicas de la realidad producidas y difundidas por los medios de comunicación de masas más masivos.

Advertimos primero que la mayoría de obras de Historia Contemporánea apenas prestaban atención a los medios de comunicación y la cultura de masas y por tanto, en palabras de Timoteo Álvarez, era necesaria "una completa reinterpretación de toda la Edad Contemporánea desde esta perspectiva, incluyendo como valor fijo la variable 'información - comunicación - propaganda'”' (Timoteo Álvarez: 1987).

Constatamos también que los historiadores que se interesaban se centraban en la prensa "de información general" en la que aparecen como protagonistas los actores 
que ocupan los escenarios públicos, medios que se dirigen a un colectivo hasta hace poco restringido a varones adultos de las clases y pueblos dominantes, del cual estuvieron excluidas las mujeres y buena parte de los hombres hasta la conformación de las democracias de masas. El resto de medios, en los que se enfoca a una mayor diversidad de seres humanos y se apela a la racionalidad y a la sentimentalidad, los más masivos, son menospreciados, igual que las mujeres y los hombres que se interesan por ellos.

Notamos, así, que el paradigma "normal” de los historiadores "olvidaba” también a las mujeres y una parte de los hombres. Y al explorar esta "anomalía” concluimos que el problema se encontraba en el concepto 'hombre', una noción presuntamente universal que, examinada con atención, delata que sólo se refiere a una parte de seres humanos, varones adultos de clases y pueblos dominantes; un colectivo que, para ejercer el poder sobre otros colectivos, asume un sistema de valores y un modelo particular de comportamiento que identificamos como un Arquetipo Viril.

Las investigaciones que realizamos (Moreno Sardà: 1986a y 1988) nos permitieron validar esta hipótesis e identificar que el pensamiento académico está gobernado por un paradigma androcéntrico construido a partir de este concepto que Aristóteles definió en la 'Politica':

Para hacer grandes obras es preciso ser tan superior como lo es el hombre a la mujer, el padre a los hijos y el amo a los esclavos.

Se trata de un modelo particular, sexista, adulto, racista y clasista, construido como si fuera superior a base de valorar como si fueran inferiores a otras mujeres y hombres, para legitimar como naturales y superiores las actuaciones viriles propias del dominio de unos colectivos sobre otros.

Esta explicación, expuesta claramente por Aristóteles, aparece opaca en el los textos académicos debido a que presenta unos fenómenos como si fueran universales a base de silenciar otros que han adquirido importancia en las sociedades contemporáneas: las actuaciones de los varones adultos de clases y pueblos dominantes en los escenarios públicos en los que ejercen el poder aparecen como las únicas significativas para examinar el funcionamiento social, mientras que las de la mayoría de mujeres y hombres en ámbitos privados y marginales aparecen como si fueran in-significantes o amenazas contra lo "normal"; además, los proyectos expansivos aparece como naturales y universales a base de no tener en cuenta las repercusiones que tiene en las relaciones sociales internas de las sociedades que los practican, en las formas de apropiación de los bienes obtenidos, en su distribución interna, privada y pública, y en cómo afecta a los colectivos a los que se empobrece para incrementar la riqueza de unas minorías.

Así, de esta conceptualización de “lo humano” se deriva un paradigma androcéntrico que impide abordar cuestiones fundamentales para comprender la implantación de los medios de comunicación de masas: su papel como sistema neurálgico de los proyectos de dominio expansivo y los rasgos específicos que han adquirido en la 
actual "era espacial"; su importancia en el ámbito público, en los espacios domésticos, personales, íntimos y los marginales; el papel de las representaciones simbólicas que difunden los medios de comunicación apelando a la racionalidad y a la sentimentalidad; en definitiva, el papel activo de la pluralidad de mujeres y hombres que tejen relaciones personales y colectivas activamente y utilizan hoy los recursos que envuelven la Tierra.

Detectar el "olvido" de las mujeres nos permitió, así, notar la pervivencia de un discurso académico propio de una institución universitaria hasta fechas recientes restringida a grupos minoritarios de varones adultos de clases y pueblos dominantes, que aunque ha abierto sus puertas a mujeres y hombres de otros grupos sociales, preserva un pensamiento obsoleto, incapaz de comprender las sociedades contemporáneas (Moreno Sardà: 2012). Y esto porque el paradigma androcéntrico expresa un "yo cognoscente" asumido por unos profesionales de las humanidades y las ciencias sociales - también por las mujeres profesionales de estos campos - que implica considerar a los seres humanos como "objetos de estudio" a los que se analiza desde una supuesta superioridad y se fragmenta según los criterios de cada especialidad, dificultando comprender la existencia humana en su unicidad.

Concluimos la necesidad de ampliar el enfoque al conjunto de mujeres y hombres, a sus actuaciones en escenarios privados, públicos y marginales, y a la diversidad de las relaciones sociales que se tejen en la actual sociedad red.

2.3. La actividad humana como actividad comunicativa: paradigmas para una historia de la comunicación no-androcéntrica

La Cibernética colaboró a resolver estos problemas. En su obra Cibernética y sociedad, Wiener (Wiener: 1965) propone comprender la sociedad a partir del estudio de los mensajes:

La sociedad solo puede ser comprendida mediante un estudio de los mensajes y de las facilidades de comunicación de que dispone y, además, en el futuro desarrollo de estos mensajes y de las facilidades de comunicación, los mensajes entre el hombre y las máquinas, entre las máquinas y el hombre y entre las mismas máquinas, están destinados a ejercer cada vez más un papel muy preponderante...

Atribuye un papel decisivo al pasado en el presente y el futuro y destaca la importancia del aprendizaje entre los seres humanos:

La especie humana es fuerte sólo en la medida en que sabe aprovechar la facultad innata y plástica de aprender y que es posible gracias a su estructura fisiológica...

Comprender la actividad humana como actividad comunicativa, la existencia humana individual y colectiva en el pasado presente y futuro como un todo unitario, que también se planteaban otros autores (Morin: 1973), nos permitió formular dos paradigmas (Moreno Sardà: 1991). 
En primer lugar, consideramos que cada ser humano es el resultado del proceso de aprendizaje de la memoria colectiva, y el funcionamiento social, producto de la asimilación o no asimilación personal de la memoria colectiva. Podemos articular así las actuaciones personales y colectivas, las historias individuales y la historia de las sociedades, relacionar las memorias personales y la memoria colectiva, percibir las pervivencias y los cambios en el proceso de reproducción generacional de la vida social, y en este marco, situar el papel de los medios de comunicación de masas como médiums que reproducen simbólicamente la realidad social.

Además, la crítica al pensamiento androcéntrico, al permitir percibir la articulación entre los fenómenos considerados significativos y los menospreciados como insignificantes, nos condujo a examinar las transformaciones de las relaciones sociales internas, privadas, públicas y marginales, teniendo en cuenta las dimensiones y las formas alcanzadas por los proyectos de dominio expansivo que se ponen de manifiesto, de forma clara, en las redes y medios de transporte y de comunicación, sistema neurálgico de estos proyectos y a la vez instrumentos que colaboran al entendimiento.

Ambos paradigmas permiten construir un nuevo relato de la historia colectiva en el que las aportaciones de cualquier persona adquieren significado y pueden colaborar a formular un humanismo plural. Este es el objetivo de la Historia de la Comunicación que definimos como no-androcéntrica, no limitada a los centros de poder sino atenta a las actuaciones de mujeres y hombres en las diversas posiciones sociales que ocupan (Haraway: 1991).

\section{De las propuestas para la innovación docente a los instrumentos para la parti- cipación ciudadana en la construcción de un humanismo plural}

Desde mediados de los años 80 desarrollamos propuestas docentes en las que pusimos a prueba estos planteamientos teóricos de la Historia de la Comunicación (Moreno Sardà: 2007).

Nos propusimos primero hacer visibles a la pluralidad de mujeres y hombres de diferentes edades, procedencias y condiciones sociales como sujetos agentes, relacionar sus itinerarios vitales con la historia colectiva, explicar la implantación de los medios de comunicación y la cultura de masas en sus historias personales, y tener en cuenta los procesos de movilidad geográfica y social: desplazar la atención de la minoría de varones adultos de clases y pueblos dominantes, privilegiados por los historiadores, para abarcar también a cualquier persona.

Tener en cuenta a otras y otros protagonistas requiere utilizar otras fuentes documentales además de las convencionales. Resultó decisiva la Historia Oral (Thompson: 1988). Las entrevistas permiten conocer las condiciones de vida de las personas desde que nacen, las actividades y relaciones en los domicilios privados, en los barrios, en las localidades donde transcurre la vida cotidiana, y examinar la incor- 
poración de los medios de transporte y de los medios de comunicación de masas no sólo como instituciones públicas, ni sólo como tecnologías, sino como instrumentos utilizados para las relaciones sociales y los procesos de movilidad. Los medios de comunicación aparecieron también como una fuente documental de gran interés para construir un relato que despierta la memoria personal y colectiva (Molina Rodríguez Navas: 2006; Molina Rodríguez Navas y otros: 2013).

En los 90 Internet planteó el reto de comprender la construcción histórica de la sociedad red: considerar cada localidad como encrucijada de tramas más amplias (Borja y Castells: 1997), tejidas históricamente mediante redes y medios de transporte y de comunicación, facilitaba ver también que cada persona teje redes de relaciones personales en la localidad en la que vive y por las que transita, desde las distintas posiciones que ocupa en su itinerario vital (Bott: 1990; González Escudero: 2000).

Los mapas aparecieron como un instrumento muy útil, y en 1998 organizamos una exposición cartográfica, Redes de Comunicación en Cataluña: pasado, presente y futuro, para disponer de material para construir la web Paseos por las redes de comunicación desde..., concebida para relacionar el pasado y el presente de cada localidad con las tramas históricas supralocales con las que se vincula mediante redes y medios de transporte y de comunicación (Moreno Sardà: 1998). La utilización docente de esta web ha permitido contrastar los planteamientos teóricos con las nuevas posibilidades que desarrolla Internet para facilitar la participación ciudadana en la construcción de conocimiento colectivo (Jiménez Chávez: 2012; Subires Mancera: 2013; ).

\subsection{Metodología}

Dado que la metodología requiere alterar rutinas del pensamiento convencionales, utilizamos la web para organizar el conocimiento de acuerdo con tres coordenadas básicas para evitar el paradigma androcéntrico dominante (https://wikis.uab.cat/ passeigperlesxarxes/index.php/LA_GU\%C3\%8DA).

La coordenada espacial permite enfocar la realidad desde cada localidad considerada como un nudo de tramas supralocales más amplias (regiones, estados, continentes, mundo), y a la inversa, aproximarse a cada espacio concreto de la vida cotidiana desde enfoques más amplios.

La coordenada temporal permite organizar el relato teniendo en cuenta que el presente es resultado de cambios y permanencias de un pasado más o menos próximo y lejano cuyas huellas se pueden identificar en los escenarios sociales locales y supralocales, y en los modelos de comportamiento que orientan las actuaciones personales en sus itinerarios vitales. La Historia de la Comunicación se plantea, así, como una arqueología de las comunicaciones.

Y los campos temáticos, están pensados para evitar incurrir en la visión androcéntrica que valora como significativo lo institucional y relega lo personal al terreno de lo insignificante, anecdótico o marginal, y poder elaborar explicaciones que permitan relacionar datos colectivos y personales. 
Técnicamente no había ningún problema; las dificultades surgían al elaborar los documentos, porque el campo temático que consideramos debe ocupar el centro de la explicación, mujeres y hombres de diferentes edades, condiciones sociales y procedencias, sus posiciones, actividades y relaciones sociales, primordial para comprender las aportaciones individuales a la historia colectiva, suele merecer una atención secundaria en las obras de los especialistas o reducirse a datos abstractos; por el contrario, la organización institucional, que se debería entender como consecuencia de la organización de las relaciones sociales, centra su atención de forma a veces exclusiva. Además, las redes y medios de transporte y de comunicación, fundamentales para identificar el sistema neurálgico que articula las vidas de las personas en localidades y tramas históricas, también recibe escasa atención en la Historia Contemporánea.

Representamos esquemáticamente la estructura básica para construir una plataforma en línea que facilite elaborar un humanismo plural, posicionado, en red y de forma cooperativa.

Esquema: estructura para construir un humanismo plural, posicionado, en red y cooperativo

\begin{tabular}{|l|l|}
\hline $\begin{array}{l}\text { Ruta para construir conocimiento colectivo } \\
\text { sobre una localidad o una trama histórica }\end{array}$ & $\begin{array}{l}\text { Ruta para organizar las aportaciones } \\
\text { personales al conocimiento colectivo }\end{array}$ \\
\hline $\begin{array}{l}\text { Documentos elaborados por personas } \\
\text { expertas sobre la época elegida, organizados } \\
\text { según campos temáticos: }\end{array}$ & $\begin{array}{l}\text { Testimonios personales (textos, fotografías, } \\
\text { otros materiales...) organizados según } \\
\text { espacios y fases de la vida en la época } \\
\text { elegida, relacionados con los campos } \\
\text { temáticos: }\end{array}$ \\
$\begin{array}{l}\text { Representación del espacio local } \\
\text { (cartografía): relación con los datos sobre el } \\
\text { conocimiento colectivo }\end{array}$ & $\begin{array}{l}\text { Testimonios sobre las condiciones } \\
\text { de nacimiento y la infancia: espacio } \\
\text { doméstico, recursos y relaciones } \\
\text { familiares, religión, escolarización... }\end{array}$ \\
$\begin{array}{l}\text { Datos sobre cantidad y distribución de la } \\
\text { población en el espacio social, acceso a los } \\
\text { recursos, organización institucional... }\end{array}$ & $\begin{array}{l}\text { Testimonios sobre experiencias, } \\
\text { expectativas, aspiraciones... en la } \\
\text { adolescencia }\end{array}$ \\
$\begin{array}{l}\text { Datos sobre Medios de transporte en el } \\
\text { interior de cada localidad y con otras } \\
\text { localidades }\end{array}$ & $\begin{array}{l}\text { Testimonios sobre decisiones en las } \\
\text { diferentes edades adultas, privadas y } \\
\text { públicas }\end{array}$ \\
$\begin{array}{l}\text { Datos sobre Medios de comunicación internos } \\
\text { y que conectan con ámbitos geográficos más } \\
\text { amplios }\end{array}$ & $\begin{array}{l}\text { Testimonio sobre las repercusiones de las } \\
\text { decisiones en las generaciones siguientes }\end{array}$ \\
$\begin{array}{l}\text { Conexión: } \rightarrow \text { Conocimiento personal } \\
\text { Conexión: } \leftarrow \text { Conocimiento colectivo local }\end{array}$ \\
\hline \multicolumn{2}{|c|}{ y de la trama histórica } \\
\hline
\end{tabular}




\subsection{Dos ejemplos}

La experiencia docente nos ha permitido plantearnos utilizar Internet para construir instrumentos para la participación ciudadana en la formulación de un humanismo plural y en la intervención política. Actualmente trabajamos en dos:

En 2007 concebimos el Mapa interactivo de los Medios de Comunicación en Cataluña (http://labcompublica.info/innovacio/mapa-de-mitjans-de-catalunya/) para elaborar una visión de los medios de comunicación distribuida territorialmente. Se trata de una infografía, que hace una representación cartográfica de Cataluña, vinculada con una base de datos en la que se registra información sobre cada medio, y que permite consultar los datos según distintos criterios. Este conocimiento se puede elaborar de forma cooperativa y en red, aunque hasta ahora esta tarea no ha resultado fácil. Constituye la estructura básica para elaborar un Atlas histórico de las comunicaciones.

También en 2007 definimos el proyecto Infoparticip@ para promover un periodismo que facilite la participación ciudadana en el control democrático (http://www. infoparticipa.cat/). De este proyecto se deriva el Mapa Infoparticip@ (www.mapainfoparticipa.com), en el que se publican los resultados de evaluar la información que proporcionan les webs de los ayuntamientos sobre quiénes son los representantes políticos y cómo gestionan los recursos colectivos a partir de 41 indicadores básicos. Los resultados de analizar cada web se traducen en una gama de colores de acuerdo con el infómetro y se georeferencian para que quien tenga interés pueda contrastarlos con la web corporativa y reclamar mejoras. Este Mapa, en el que colaboran actualmente investigadoras e investigadores de 9 universidades de 6 comunidades autónomas, con financiación del MINECO (CSO2012-34687), está colaborando a promover la calidad y la transparencia de la comunicación pública local.

\section{Conclusiones}

Internet facilita hoy la participación ciudadana en la construcción de conocimiento colectivo. La Historia de la Comunicación tal como la hemos formulado demuestra que los problemas técnicos se pueden resolver, pero que la dificultad estriba en el paradigma académico dominante, androcéntrico, restrictivo, vertical, asumido por los profesionales, que dificulta que las aportaciones especializadas se acoplen con las aportaciones personales para construir un nuevo conocimiento colectivo, e impide que las intervenciones de mujeres y hombres se tengan en cuenta como datos significativos para formular el humanismo plural que hoy necesitamos para comprender la sociedad en la que vivimos e intervenir para hacerla más justa. De ahí la necesaria revolución de las Ciencias Humanas y Sociales. 


\section{Bibliografía}

BARRACLOUG, G. (1965), Introducción a la Historia Contemporánea, Madrid: Gredos

BORJA, J., CASTELLS, M. (1997): Local y global. La gestión de las ciudades en la era de la información. Madrid: Taurus.

BOTT, E. (1990), Familia y red social. Madrid: Taurus.

CASTELLS, M. (2009), Comunicación y poder. Madrid: Alianza

CROWLEY, P; HEYER, P. (ed.) (1997), La Comunicación en la Historia, Tecnología, Cultura, Sociedad, Barcelona: Bosch.

FLICHY, P. (1991), Una historia de la comunicación moderna. Espacio público y vida privada. Barcelona: Gustavo Gili.

GASCON MARTÍN, F. (2003), Transformaciones sociales, redes y politicas de comunicación en Chile (1967-2001). Elementos para una ecología política de las comunicaciones. Tesis Doctoral. Departamento de Periodismo y Ciencias de la Comunicación. Universidad Autónoma de Barcelona: http://www.tdx.cat/ handle/10803/4174 [15-III-2012].

GONZÁLEZ ESCUDERO, E. (2000), Redes sociales, comunicación y procesos de movilidad y asentamiento de los emigrantes magrebies en Alicante (19851995). Tesis Doctoral. Departamento de Periodismo y Ciencias de la Comunicación, Universidad Autónoma de Barcelona. Disponible en: http://www.tdx.cat/ handle/10803/4173 [15-III-2012].

HARAWAY, D. J. (1995): Ciencia, cyborgs y mujeres. La reinvención de la naturaleza. Madrid: Cátedra.

KENNEDY, P. (2006), Auge y caída de las grandes potencias, Madrid: Debolsillo.

KUHN, T. S. (1982), La estructura de las revoluciones cientificas, Madrid: Fondo de Cultura Económica.

McBRIDE et alt. (1980), Un solo mundo, voces múltiples. Comunicación e información en nuestro tiempo, México: FCE.

McNEILL, J. R.; McNEILL, W. H. (2010), Las redes humanas. Una historia global del mundo, Barcelona: Crítica

MOLINA RODRÍGUEZ NAVAS, P. (2006), Encontrarse en la historia. Fuentes, protagonistas y espacios: metodologías para una historia de la comunicación no-androcéntrica. Tesis Doctoral. Departamento de Periodismo y Ciencias de la Comunicación. Universidad Autónoma de Barcelona. Disponible en: http://www. tdx.cat/handle/10803/4198 [15-III-2012].

MORENO SARDÀ, A. (1986a), El Arquetipo Viril protagonista de la historia. Ejercicios de lectura no androcéntrica. Barcelona: LaSal, Edicions de les dones. (1986b), "El surgimiento de la prensa de masas". En FONTCUBERTA, M. de (dir.): Enciclopedia del Periodismo, vol. 3, El periodismo escrito. Barcelona: Mitre, p. 7-13

(1988), La otra 'Politica' de Aristóteles. Cultura de masas y divulgación del arquetipo viril. Barcelona: Icaria.

(1991), Pensar la historia a ras de piel, Barcelona: Ediciones de la Tempestad. 
(1997), "Presentación de la edición en castellano". En CROWLEY Y HEYER (ed.), (1997), La Comunicación en la Historia, Tecnología, Cultura, Sociedad, Bosch, Barcelona, páginas 7-11.

(2007), De qué hablamos cuando hablamos del hombre. Treinta años de crítica y alternativas al pensamiento androcéntrico. Barcelona: Icaria

(2012), "Aportaciones que podemos hacer las mujeres a la revolución de las Ciencias Humanas y Sociales para construir un humanismo plural en la era digital". En CORTÉS, C., MORALES, I. M ${ }^{\mathrm{a}}$, y VERA, $\mathrm{M}^{\mathrm{a}} \mathrm{T}$. (coord.), I Jornadas Universitarias del Posgrado sobre Igualdad y Género. Actas de Ponencias y Resúmenes, Málaga: Del Planeta Rojo Ediciones, p. 13-31.

MORIN, E. (1973), El paradigma perdido: el paraíso olvidado. Ensayo de bioantropología. Barcelona: Kairós.

ROWLAND JR., W. D. (1997) "Prefacio". En CROWLEY Y HEYER (ed.) (1997), La Comunicación en la Historia ..., p. 13.

SUBIRES MANCERA, M. P. (2013), Tic, sociedad y territorio: Internet como medio de comunicación, información y gestión del conocimiento para la dinamización en el ámbito rural. La experiencia de los Centros Guadalinfo. Valle del Guadalhorce y Sierra de las Nieves (2006-2013). Tesis Doctoral. Departamento de Periodismo. Universidad de Málaga.

THOMPSON, P. (1988), La voz del pasado. Historia oral. Valencia: Edicions Alfons el Magnànim.

TIMOTEO ÁLVAREZ, J. (1987), Historia y modelos de la comunicación en el siglo $X X$. El nuevo orden informativo, Barcelona: Ariel WIENER, N. (1965), Cibernètica i societat, Barcelona: Edicions 62.

\section{La autora}

Amparo Moreno Sardà, catedrática emérita de la Facultad de Ciencias de la Comunicación de la Universidad Autónoma de Barcelona, ha impartido Historia de la Comunicación, Métodos de Investigación y Comunicación Local. Su investigación inicial sobre prensa de sucesos le condujo a formular la hipótesis de que el arquetipo viril y el androcentrismo del pensamiento académico dificultan comprender las sociedades contemporáneas, y a elaborar nuevos paradigmas y propuestas docentes para abarcar la pluralidad de mujeres y hombres y medios de comunicación. Internet le ha permitido aplicar estos planteamientos elaborar productos para la renovación periodística con el equipo que dirige, el LPCCP de la UAB (http://www.labcompublica. org/). 